\title{
Cold atoms: A new medium for quantum optics
}

\author{
A. Lambrecht, J.M. Courty, S. Reynaud, E. Giacobino \\ Laboratoire Kastler Brossel, UPMC, ENS, CNRS \\ Université Pierre et Marie Curie, 4 place Jussieu, F 75252 PARIS \\ (Applied Physics B60, p.129 (1995))
}

\begin{abstract}
Laser cooled and trapped cesium atoms have been used as a non-linear medium in a nearly resonant cavity. A study of the semi-classical dynamics of the system was performed, showing bistability and instabilities. In the quantum domain, squeezing in a probe beam having interacted with this system was demonstrated.
\end{abstract}

PACS: 32.80.Bx; 32.80.Pj; 42.50.Dv; 42.50.Lc; 42.65.Pc

Cold atoms in a magneto-optical trap constitute a quite new non-linear material, the properties of which are far from being fully investigated. With the very low temperatures that can be achieved, the atoms can be considered as virtually motionless and the Doppler width of their resonances is smaller than the natural linewidth. In the vicinity of resonance lines, one can expect large non-linear dispersive effects associated with very little absorption, but it is only recently that a cloud of cold atoms was placed in a resonant optical cavity to study its semi-classical dynamics [1]. As we show in the first part of this paper, cold cesium atoms in a cavity have a very rich non-linear dynamics, exhibiting not only bistability but also instabilities. Analysing the non-linear dynamics of laser cooled atoms in a cavity was a prerequisite to the investigation of quantum noise in order to find the appropriate conditions to study the quantum properties of a light beam going out of the cavity.

The generation of quadrature squeezed light through interaction with atomic media has been the subject of numerous theoretical studies and looked particularly promising at the condition that there is no Doppler effect. All the experiments up to date were performed on atomic beams. In the second part of this paper, we report on the quantum properties of cold atoms in a cavity and show that it is possible to detect quadrature squeezing in a probe beam that has passed through the cavity.

\section{DYNAMICS OF COLD ATOMS IN A CAVITY}

We investigate the dynamical non-linear behaviour of an optical cavity containing cold cesium atoms by sending a circularly polarisedprobe beam into the cavity. The atoms are cooled and trapped in a magneto-optical trap, operating in the standard way [2] with three orthogonal circularly polarised trapping beams generated by a Ti:Sapphire laser and an inhomogeneous magnetic field.

The cavity is a $25 \mathrm{~cm}$ long linear asymmetrical cavity, close to half-confocal, with a waist of $260 \mu \mathrm{m}$, built around the cell (Fig. 1). Losses due to the two AR coated windows are of the order of $1 \%$. The input mirror has a transmission coefficient of $10 \%$, the end mirror is highly reflecting, which gives a cavity linewidth of $5 \mathrm{MHz}$. The cavity is in the symmetry plane of the trap, making a $45^{\circ}$ angle with the two trapping beams that propagate in this plane.

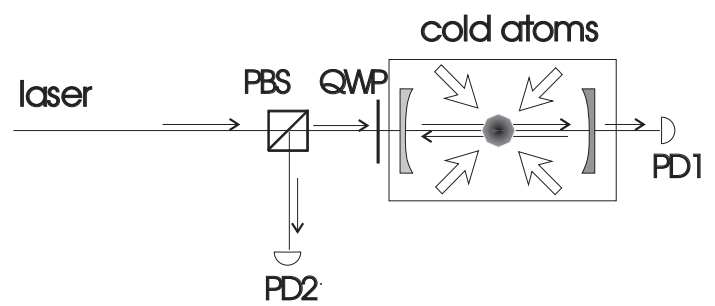

FIG. 1. Experimental set-up showing the cell containing the cold atom cloud in an optical cavity; PBS: polarising beamsplitter, QWP: quarter-wave plate, PD1, PD2: photodiodes; PD1 and PD2 measure the powers respectively transmitted and reflected by the cavity.

The trapping beams are detuned by 2.5 times the linewidth of the upper state $(\Gamma / 2 \pi=5.2 \mathrm{MHz})$ on the low frequency side of the $6 S_{1 / 2} F=4$ to $6 P_{3 / 2} F=5$ transition, yielding a cloud of cesium atoms the typical temperature of which is of the order of $1 \mathrm{mK}$. The diameter $(2.5 \mathrm{~cm})$ and power $\left(20 \mathrm{~mW} / \mathrm{cm}^{2}\right)$ of the trapping to obtain large clouds (about $8 \mathrm{~mm}$ in diameter) with densities of the order of beams allow us $10^{10}$ atoms $/ \mathrm{cm}^{3}$. The number of trapped atoms located in the probe beam, which is the relevant parameter in the experiment, measured from the change in the transmission of the cavity in the presence of atoms, is found to be ranging between $10^{7}$ and $10^{8}$ depending on the pressure of the background gas. As usual, the atoms non resonantly excited into the $6 P_{3 / 2} F=4$ state and falling back into the $F=3$ ground state are repumped into the cooling cycle by a laser diode tuned to the $6 S_{1 / 2} F=3$ to $6 P_{3 / 2} F=4$ transition.

The probe beam, generated by the same laser, can be detuned by 0 to $130 \mathrm{MHz}$ (corresponding to $0-25 \Gamma$ ) on either side of the $6 S_{1 / 2} F=4$ to $6 P_{3 / 2} F=5$ atomic transition. We measure the power of the probe beam transmitted through the cavity while scanning the cavity length for a fixed value of the input intensity as shown in Fig. 2, or the input intensity for a fixed value of the detuning. The recording shows the characteristic hysteresis cycle due to bistability, where the output power switches abruptly between low and high values when the length of the cavity is scanned. 
transmitted power (a.u.)

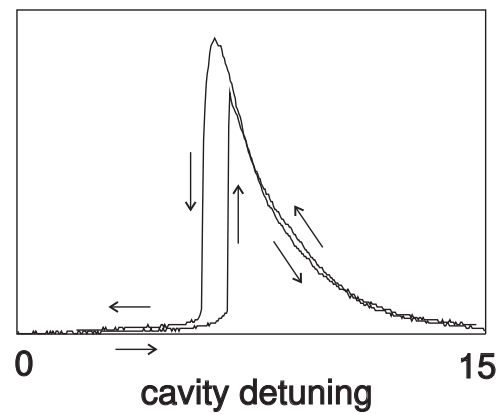

FIG. 2. Recording showing the bistable switching from low to high transmission and back when the cavity length is scanned across resonance. The trapping beams are on. The probe laser is detuned by $22 \Gamma$ on the high frequency side of the atomic transition; the input power is $100 \mu \mathrm{W}$. Cavity detuning is in units of the cavity linewidth $\gamma_{\text {cav }} / 2 \pi \tau$.

The shape of the curves is compatible with the theory of bistability with two-level atoms. However, the $F=4$ to $F=5$ transition under investigation is far from being a two-level one. In the presence of the trapping beams, it can be approximately described as a set of two-level systems: the circularly polarised probe beam interacts with all $m_{F}$ to $m_{F+1}$ transitions from the ground to excited state, while the trapping beams randomise the ground state population among the various Zeeman sublevels. In this sense the bistability curve shown in Fig. 2 has to be understood as an effective bistability, in which all sublevels participate.

When the probe beam is strong enough, the induced optical pumping cannot be neglected. To thoroughly investigate the dynamics of the system, it was better to avoid a competition between trapping beams and probe beam in the interaction with the atoms. Consequently, the trapping laser beams are turned off after the trap has been loaded. The time left for the measurement is about $20 \mathrm{~ms}$ before most of the atoms have escaped out of the interaction region due to free fall and expansion of the cloud. A significant measure of the number of atoms consists in the bistability parameter $C$, which is the ratio of linear absorption of the atoms at resonance to the energy transmission coefficient of the cavity. Right after the atoms have been released, the bistability parameter can be as high as 400 .

In a broad range of experimental parameters, not only bistability but instabilities are observed. Figure 3 shows a recording of the transmitted power of the cavity for two different values of the input power, while the cavity length is scanned. At low input powers of the order of $50 \mu \mathrm{W}$ oscillations appear on the left hand side of the cavity resonance. This side is the one on which bistable switching would occur in a saturated two-level atomic system. In particular it has to be noted that the first trace (input power $100 \mu \mathrm{W}$ ) was recorded under the same experimental conditions as the trace in Fig. 2, except that the trapping beams were switched off. While in presence of the beams the system is clearly bistable, in their absence instabilities appear instead. At high input powers (about $300 \mu \mathrm{W}$ ) the oscillations disappear and only bistability persists.

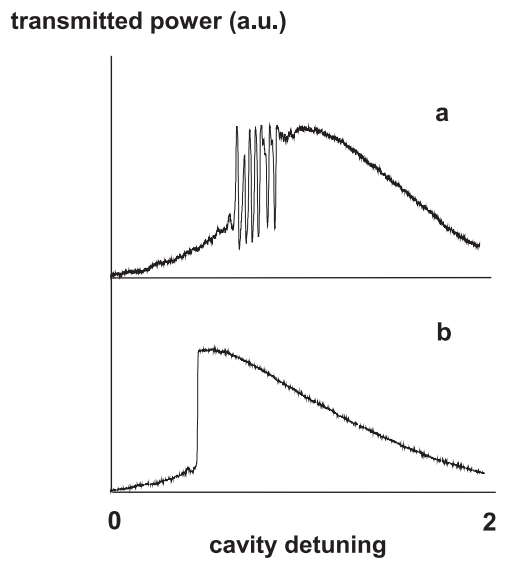

FIG. 3. Recording of the power transmitted through the cavity containing cold atoms while the cavity length is scanned across resonance. Input powers are $P_{\text {in }}=100 \mu \mathrm{W}$ (a) and $\mathrm{P}_{\text {in }}=350 \mu \mathrm{W}$ (b) respectively. The atomic detuning is $22 \Gamma$ on the blue side of the atomic transition, the trapping beams are off. Cavity detuning is in units of the cavity linewidth $\gamma_{\text {cav }} / 2 \pi \tau$.

Instabilities are present within the whole range of accessible detunings on either side of the resonance. Their frequency is comprised between $100 \mathrm{kHz}$ and a few $\mathrm{MHz}$. They can also be observed when the cavity length is scanned by the escape of the atoms from the original cloud, which changes the index of refraction.

To understand these oscillations, the hyperfine and Zeeman structure of the considered states and the various possible optical pumping effects have to be taken into account. Instabilities 3,4.5 linked to optical pumping were predicted and observed in alkali vapours. However, this system is rather intricate to describe, due to the interaction of the field with atoms in various velocity classes and hyperfine sublevels.

The situation is much simpler with cold atoms, where one can consider that the field interacts with one hyperfine transition only. Owing to this, we have been able to understand the origin of the observed oscillations with a simple model. Roughly, as shown below, they result from the competition between two non-linear processes, saturation of the optical transition, which tends to decrease the linear index of refraction of the atomic medium when the intensity increases, and optical pumping that increases the index of refraction by accumulating the atoms in magnetic sublevels with a high $m_{F}$ number, which have the largest coupling coefficient with the light.

While the saturation of the optical transition is virtually instantaneous, it takes a comparably long time to complete the optical pumping to the highest $\mathrm{mF}$ sublevels of the ground and excited states, starting from an equally distributed population in the ground state. Even 
for intensities close to or larger than the saturation intensity the optical pumping rate is much smaller than the natural linewidth [6]. The relaxation oscillations are a consequence of the significant difference in the characteristic times of these processes.

Although the behaviour of the system involving many variables (all hyperfine Zeeman populations and coherences and the field) is quite complex, the underlying mechanism can be explained with a simple model, based on essentially two differential equations, one for the evolution of the intracavity field and the other for the atomic orientation in the ground state.

The change of the intracavity field a on a round trip of time duration $\tau$ is due to the driving field $\alpha_{\text {in }}$ entering through the coupling mirror of transmission $t$, to the mirror decay coefficient gcav (with $\gamma_{\text {cav }}=t^{2} / 2$ ) and to the round trip phase shift $\phi_{\text {cav }}$ :

$$
\tau \frac{\mathrm{d} \alpha}{\mathrm{d} t}=t \alpha_{\mathrm{in}}-\left(\gamma_{\mathrm{cav}}-i \phi_{\mathrm{cav}}\right) \alpha
$$

The total phase shift in the cavity $\phi_{\text {cav }}$ writes:

$$
\phi_{\mathrm{cav}}=\phi_{0}+\phi_{\mathrm{L}}(1+p)+\phi_{\mathrm{NL}}
$$

where $\phi_{0}$ is the round trip phase shift in the empty cavity, $\phi_{L}$ is the term due to the linear index of the atoms and $p \phi_{L}$ the additional contribution associated with the ground state orientation of the atoms induced by optical pumping; $\phi_{N L}$ is the Kerr-like phase shift due to saturation of two-level atoms.

The ground state orientation $\mathrm{p}$ increases with the intracavity intensity $I$ at a rate $\beta I$ and decays at a rate $\gamma_{p}$ by magnetic precession in transverse fields and by transitions to other hyperfine sublevels (via non resonant transitions):

$$
\frac{\mathrm{d} p}{\mathrm{~d} t}=-\gamma_{\mathrm{p}} p+\beta I(1-p)
$$

In the presence of optical pumping equations (11) and (3) have to be solved numerically. In some range of initial conditions, oscillations and limit cycles are found in the intracavity intensity as well as in the output intensity. The calculated output intensity as a function of the cavity length is shown in Fig. 4 for both a low and a high value of the input intensity. It can be seen that the curves reproduce the experimental recordings in a satisfactory way. Oscillations are essentially manifest when the different time constants involved are such, that (already small) variations in the atomic ground state orientation scan the cavity back and forth around the bistable switching point and therefore lead to periodic abrupt changes in the intracavity power. Only at high input powers instabilities disappear, as the optical pumping is fast enough to bring all atoms in the sublevel with the highest magnetic number before the oscillations can start. In this sense also the absence of the instabilities in presence of the trapping beams (Fig. 2) can now be understood, because optical pumping by the probe beam is counteracted by the trapping beams, which themselves produce a strong optical pumping of the atoms, which tends to randomise the populations in the various sublevels.

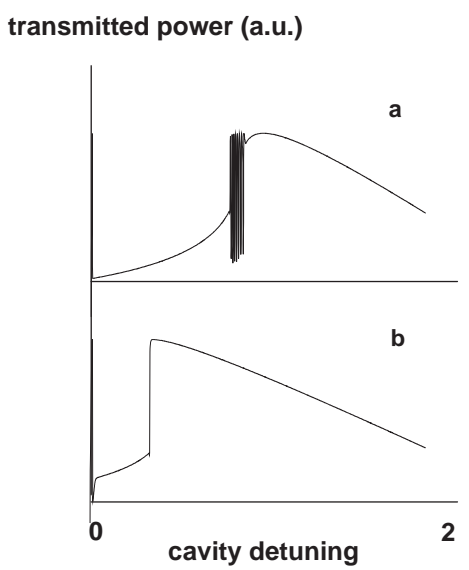

FIG. 4. Calculated power transmitted through the cavity containing cold atoms as a function of cavity detuning (normalised to the cavity linewidth $\gamma_{\text {cav }} / 2 \pi \tau$ ). Input powers correspond respectively to 1.3 (a) and 2 (b) times the bistability threshold power in the absence of optical pumping.

The validity of the model was checked by further experiments. When the atoms are released from the trap, it is possible to optically pump them into the $m_{F}=4$ magnetic sublevel of the ground state with an additional circularly polarised beam parallel to the probe beam, but closer to the atomic resonance. This prepumping is done in the presence of a magnetic field directed along the cavity. In such conditions the instabilities disappear. If the magnetic field is absent, the orientation created by the pump field is destroyed by the Larmor precession in transverse magnetic fields and the instabilities persist.

A more complete theoretical treatment of the atomic non-linearity and of the optical pumping was also performed, where absorption and saturation of the optical non-linearity were taken into account. The general behaviour remains the same as the one predicted by the simple model.

If cold atoms are to be used as a non-linear medium to produce squeezed light, clearly instabilities have to be avoided. The results presented so far show that, although the behaviour of the system is more complicated than anticipated, it is possible to find conditions where cold cesium atoms behave as two-level atoms.

\section{QUANTUM NOISE MEASUREMENTS}

Nearly perfect squeezing is expected from a cavity containing a pure Kerr medium [7, 8, 9, When motionless two-level atoms are used, the quantum noise reduction is predicted to persist in spite of added fluorescence noise. Specific theories have to be used to take these effects 
into account [10,11, 12,13]. Squeezed vacuum was first observed in a four-wave mixing experiment using an atomic beam as a non-linear medium [14. Later, squeezing in a beam of finite mean intensity (bright squeezing) was demonstrated at the output of a bistable cavity traversed by an atomic beam [15, 16.

Cold atoms seem to be good candidates to achieve quantum noise reduction, since their motion is much better controlled than in an atomic beam. However, observation of squeezing requires that the trapping beams are absent. Otherwise a part of the atoms would be constantly excited to the $6 P_{3 / 2}$ state and fluoresce back to the ground state, causing excess noise. This condition imposed the study of the non-linear dynamics of the cavity in absence of the trapping beams. To avoid excess noise and instabilities both trapping beams and magnetic field gradient are therefore turned off during the noise measurement; residual transverse fields are largely compensated by a homogeneous magnetic field.

The experimental set-up used to study the quantum fluctuations of a probe beam after interaction with the system is shown in Fig. 5. The linear cavity containing the atom cloud was described in section 1 . It has only one coupling mirror of $10 \%$ transmission, so as to be close to the "bad cavity" case, where the damping rate $\gamma_{\text {cav }} / \tau$ of the cavity is larger than the one of the atomic polarisation $\Gamma / 2$, and for which the theoretically predicted squeezing is the best. The second mirror is a highly reflecting one. The mode matching efficiency of the cavity with the input beam is of the order of at least $96 \%$ in power.

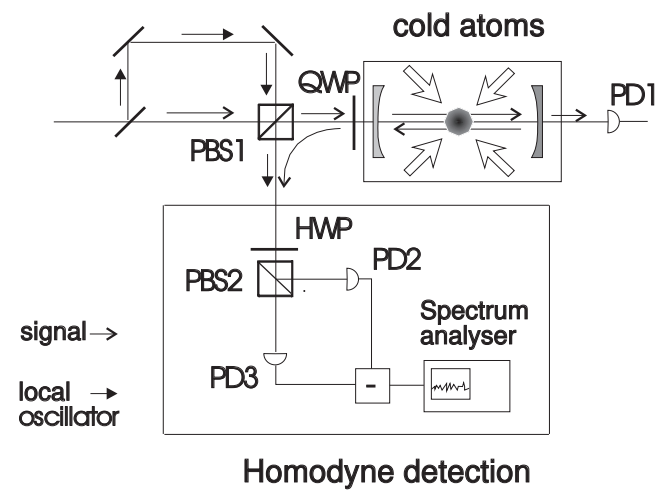

FIG. 5. Experimental layout to measure the quantum noise of a probe beam having passed through the cavity. PBS1, PBS2: polarising beamsplitters, QWP: quarter-wave plate, HWP: half-wave plate, PD1, PD2, PD3: photodiodes; PD1 measures the transmitted power; PD2 and PD3 are employed in the homodyne detection to record the reflected power and the quantum noise.

As already mentioned, the probe laser beam is generated by the same Ti:Sapphire laser as the trapping beams. This laser has the main advantage over diode lasers that it is free from any excess noise in the $\mathrm{MHz}$ range. However, this imposes to adjust the frequency of the various beams with the help of acousto-optic modulators. The only beam which is not frequency-shifted is the probe beam, in order to avoid excess noise produced by the AOM.

In the presence of the trap, the atom cloud is carefully centred on the probe beam by maximising the emission of the "cold atom Raman laser" [17, which spontaneously oscillates even in the absence of a probe beam when the cavity is well aligned around the cloud.

The probe beam coming out of the cavity is separated from the input beam by an optical circulator, made of a polarising beamsplitter and a quarter-wave plate, and mixed with a local oscillator, 100 times more intense than the probe beam, using the second input port of the same beamsplitter. At the output of the beamsplitter, the signal and the local oscillator beams are perfectly aligned and have orthogonal polarisations. A half-wave plate and a second polarising beamsplitter are used to split the combined beam of signal and local oscillator into two beams of equal power, which are then detected by two identical EG\&G FFD-100 photodiodes, operated with a bias voltage of $80 \mathrm{~V}$. The quantum efficiency of the photodiodes was found to be $86 \%$; it was further increased to $94 \%$ by the method of refocusing the reflected light. The low frequency components of photocurrents are dc coupled to provide a direct measurement of the mean power. The high frequency components are ac coupled, amplified and subtracted to monitor the quantum noise of the probe beam. The common mode rejection generally achieved in this balanced homodyne detection is better than $20 \mathrm{~dB}$ in the range of 0 to $20 \mathrm{MHz}$. The noise signal is further amplified, sent to a spectrum analyser and recorded in a digital storage oscilloscope. The mode matching between the signal and the local oscillator is optimised by measuring the dc visibility of the interference fringes on one detector when the phase of the local oscillator is scanned. The efficiency of mode matching at resonance is usually found to be of the order of $90 \%$. The total homodyne efficiency varies between $85 \%$ at resonance and $90 \%$ on the sides of the resonance curve.

The fluctuations of the field are detected at a fixed analysis frequency $\Omega / 2 \pi$, while the phase of the local oscillator is rapidly varied with a piezoelectric transducer and the length of the cavity is scanned across resonance by the escape of the atoms after the trap is turned off. When the cavity is close to resonance, the presence of the non-linear medium manifests itself by a phase dependent noise.

Detection of squeezing during the rather narrow time slot in which the atoms stay inside the beam is a non trivial problem. Having only 20-30 milliseconds to perform the measurement, important variations like the phase scan of the local oscillator have to take place at frequencies of the order of $\mathrm{kHz}$. To record a spectrum, the bandwidth of the spectrum analyser has consequently to be chosen equal to or larger than $100 \mathrm{kHz}$. On the other hand squeezing is quite sensitive to the observation frequency, thus a bandwidth larger than $1 \mathrm{MHz}$ has to be avoided not to average out the effect. A less obvious difficulty consists in the use of the so called videofilter, 
which is usually a simple postdetection low-pass filter, acting only on the display of the analysed signal to extract the essential information, which would otherwise be covered by high frequency noise. Its bandwidth is usually chosen to be $1 / 300$ of the analysing bandwidth, i.e. $300 \mathrm{~Hz}$ for a bandwidth of $100 \mathrm{kHz}$.

The signal to be analysed, that is the noise power of the light beam, is a sine function having a fundamental frequency of the order of $1 \mathrm{kHz}$. As a consequence a linear (amplitude) or logarithmic (dB) display will not only involve the fundamental frequency, but also higher harmonics. In particular in Fourier space, an important part of information about the squeezing will be stored in higher harmonics and the better the squeezing is the more important higher harmonics are. Being displayed on a linear scale, a signal containing up to $50 \%$ squeezing in power, will contain no visible squeezing anymore, if a postdetection video bandwidth of $300 \mathrm{~Hz}$ is used. We thus had to implement better adapted linear filters than a spectrum analyser can supply, which means numerical filters.

The spectra should then be recorded without videofilter at all, converted into noise power and filtered afterwards by a numerical RC filter at a well adapted frequency to reduce the high frequency noise. As we know exactly the frequency response of the videofilter, it is also possible to record a filtered signal, reconstruct the original one numerically in the frequency domain and to filter this signal converted into noise power with a numerical low-pass at a better adapted frequency. The numerical filters were compared to usual electrical RC filters; the effect on the spectrum was found to be the same. They are thus equivalent to standard postdetection videofilters, but have the essential advantage that their RC time can be freely chosen.

Figure 6 shows a spectrum reconstructed from a filtered one using the procedure above described. It displays a recording of the noise power obtained at a fixed geometrical cavity length while the changing number of atoms is scanning the cavity across resonance. Out of resonance (on the left hand side of the figure) the probe beam is totally reflected by the cavity and is hence at shot noise. The average shot noise level is indicated by the straight line. When the cavity becomes resonant with the probe beam, the noise exhibits large oscillations due to the phase scan of the local oscillator, which indicate the presence of phase dependent fluctuations. On the lower branch of the bistability curve, i.e. before the system switches towards high intensities, a quantum noise reduction of about $30 \%$ within $\pm 10 \%$ can be seen, whereas on the upper branch large excess noise is observed in some quadratures. If one takes into account losses in propagation, detection and matching, a value of the squeezing better by $4 \%$ can be inferred. This trace was obtained at an observation frequency of $\Omega / 2 \pi=5 \mathrm{MHz}$. The cavity linewidth $\left(\gamma_{\mathrm{cav}} / 2 \pi \tau=5 \mathrm{MHz}\right)$ was about as large as the atomic linewidth. Probe laser and local oscillator were detuned by $10 \Gamma$ on the high frequency side of the atomic resonance and their powers were $25 \mu \mathrm{W}$ and $9 \mathrm{~mW}$ respectively. The bistability parameter $C$ was found to be about 300 right after turning off the trapping beams.

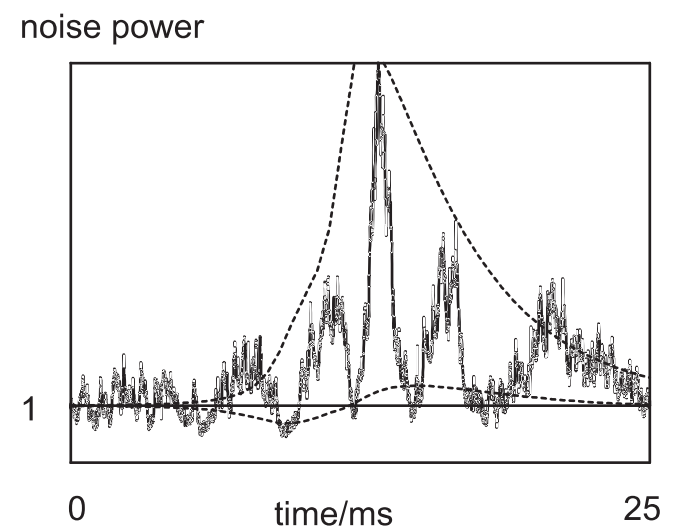

FIG. 6. Recording of the quantum noise power at $\Omega / 2 \pi=5 \mathrm{MHz}$ as a function of time, while the decaying number of atoms is scanning the cavity across resonance. The probe beam fluctuations drop below shot noise on the lower branch of the bistability curve (left hand side of trace). Probe beam and local oscillator were detuned by $10 \Gamma$ to the red side of the atomic resonance. Their powers were $25 \mu \mathrm{W}$ and $9 \mathrm{~mW}$ respectively. The straight line indicates the average shot noise level; the broken lines correspond to theoretical predictions of the minimal and maximal noise power using the experimental data. The experimental trace was taken with a postdetection video bandwidth of $300 \mathrm{~Hz}$, reconstructed numerically, converted into noise power and low pass filtered at $1 \mathrm{kHz}$ afterwards.

This result has been compared with theoretical predictions given by the two-level atom model derived from [12] including the experimental parameters. This model was developed within the plane-wave approximation and uses a linear input-output formalism to calculate the modifications of quantum fluctuations of a coherent field after interaction with an optical ring cavity containing an ensemble of two-level atoms. In agreement with the experimental situation the cavity is scanned at a fixed geometrical length by the change in the number of atoms interacting with the probe beam. In a first approximation the relevant number of atoms is assumed to decay exponentially in time. The quantum noise power in all quadratures can then be calculated as a function of number of atoms inside the probe beam and thus as a function of time. The theoretical curves shown as broken lines in Figure 6 have been computed for the experimental data given above, supposing an initial bistability parameter of 300. The two traces correspond to the minimal and maximal noise power respectively and are in reasonable agreement with the experiment. The next experimental step will consist in finding the best conditions for squeezing by exploring carefully the parameter space. On the theoretical side a more exact treatment of the interaction, including in particular the temporal variation of the number of atoms and the effect of a radially varying 
electric field, will be carried out.

\section{CONCLUSION}

Our study shows that cold atoms constitute a promising medium for non-linear and quantum optics. The fact that it is possible to generate squeezed light placing this medium inside an optical cavity opens the way to quantum optics using cold atoms and hence provides an interesting alternative to atomic beams. One of the difficulties of this experiment, coming from the fact that the trapping beams have to be turned off during the measurements, should be possible to circumvent in the future by working on a transition not coupled to the trapping transition or by using a "dark SPOT" [18] demonstrated recently.

Acknowledgements: The authors sincerely thank A. Heidmann for his very valuable help. This work has been supported in part by the EC contract ESPRIT BRA 6934 and the EC HMC contract CHRX-CT 930114.

[1] E. Giacobino, J.M. Courty, C. Fabre, L. Hilico, A. Lambrecht, in "Laser Spectroscopy IX" eds L.Bloomfield, T. Gallagher, D. Larson, AIP Conference Proceedings (AIP, New York, 1993) 290, p. 349

[2] E. Raab, M. Prentiss, A. Cable, S. Chu, D. Pritchard, Phys. Rev. Lett. 59 (1987) 2631; C. Monroe, W. Swann, H. Robinson, C. Wiemann, Phys. Rev. Lett. 65, 1571 (1990)

[3] G. Giusfredi, S. Cecchi, E. Petriella, P. Salieri and F.T. Arecchi, in "Instabilities and Chaos in Quantum Optics" eds F.T. Arecchi and R.G. Harrison (Springer, Berlin, Heidelberg 1987)

[4] P. La Penna and G. Giusfredi, Phys. Rev. A 48, 2299 (1993)

[5] F. Mitschke, J. Mlynek and W. Lange, Phys. Rev.Lett. 50, 1660 (1983)

[6] E. Giacobino, J.M. Courty, A. Lambrecht in "Quantum Optics", Springer Proceedings in Physics, eds D.F. Walls and D.J. Harvey (Springer Berlin, Heidelberg 1994); A. Lambrecht, E. Giacobino, J.M. Courty: Opt. Commun. (in press)

[7] L. Lugiato, G. Strini, Optics Commun. 41, 67 and 374 (1982)

[8] M.J. Collett, D.F. Walls, Phys. Rev. A 32, 2887 (1985)

[9] S. Reynaud, C. Fabre, E. Giacobino, A. Heidmann, Phys. Rev. A 40, 1440 (1989)

[10] L.A. Orozco, M.G. Raizen, Min Xiao, R.J. Brecha, H.J. Kimble, J. Opt. Soc. Am. B 4, 1490 (1987)

[11] F. Castelli, L.A. Lugiato, M. Vadacchino, Nuovo Cimento B 10, 183 (1988)

[12] M.D. Reid, Phys. Rev. A 37, 4792 (1988)

[13] L. Hilico, C. Fabre, S. Reynaud; E. Giacobino, Phys. Rev. A 46, 4397 (1992)
[14] R.E. Slusher, L. Hollberg, B. Yurke, J. Mertz, J.F. Valley, Phys. Rev. Letters 55, 2409 (1985)

[15] M.G. Raizen, L.A. Orozco, Min Xiao, T.L. Boyd, H. J. Kimble, Phys. Rev. Lett. 59, 198 (1987)

[16] D.M. Hope, H.A. Bachor, P.J. Manson, D.E. McClelland, P.T.H. Fisk, Phys. Rev. A 46, R1181, (1992)

[17] L. Hilico, C. Fabre, E. Giacobino, Europhys. Lett. 18, 685 (1992)

[18] Ketterle, K.B. Davis, M.A. Joffe, A. Martin, D.E. Pritchard, Phys. Rev. Lett. 70, 2253 (1993) 